SCIENCE CHINA

Physics, Mechanics \& Astronomy

\title{
Hypersonic I-shaped aerodynamic configurations
}

\author{
Kai Cui ${ }^{1,2 *}$, Yao Xiao ${ }^{1,2}$, YingZhou $\mathrm{Xu}^{1,2}$, and GuangLi $\mathrm{Li}^{1,2^{*}}$ \\ ${ }^{1}$ Key Laboratory of High Temperature Gas Dynamics of Institute of Mechanics, Chinese Academy of Sciences, Beijing 100190, China, \\ ${ }^{2}$ School of Engineering Science, University of Chinese Academy of Sciences, Beijing 100049, China
}

Received August 30, 2017; accepted October 11, 2017; published online December 18, 2017

Citation: K. Cui, Y. Xiao, Y. Z. Xu, and G. L. Li, Hypersonic I-shaped aerodynamic configurations, Sci. China-Phys. Mech. Astron. 61, 024722 (2018), https://doi.org/10.1007/s11433-017-9117-8

Hypersonic vehicles, which flight at Mach numbers greater than 5, will serve as a more convenient and efficient transport tool than present subsonic airplanes for long-distance journeys in future. Typically, it only takes a couple of hours from Beijing to New York at hypersonic speed. Recent interest in these vehicles has grown intensively, and various types of innovative designs have been proposed and studied. Despite entering the age of hypersonic flight, there still exist many problems to resolve [1-4]. How to design an advanced aerodynamic configuration is one of them [5].

In general, there are at least three objectives in designing an aerodynamic configuration, the high lift to drag ratio $(L / D)$, the high volumetric efficiency and the high lift coefficient [6]. Designers always take the high $L / D$ during the cruise state as the primary goal because the flight range is linearly proportional to the $L / D$ according to the Breguet's equation. In addition, a vehicle should provide sufficient space to contain equipments, passengers and cargoes as many as possible. Furthermore, the aerodynamic lift coefficient should be enhanced in whatever way possible. This is because a vehicle with high lift may elevate the vehicle to a high altitude where the aero-thermal environment is efficiently improved owing to the low atmosphere density.

Unfortunately, it is difficult to obtain a high $L / D$ for a generic configuration due to the presence of strong shock wave drag and massive viscosity in the hypersonic regime. Moreover, there exist strong contradictions among the $L / D$,

*Corresponding authors (Kai Cui, email: kcui@imech.ac.cn; GuangLi Li, email: liguangli11@mails.ucas.ac.cn) the volumetric efficiency and the lift coefficient [7]. Among the existing configurations, the waverider has been deemed the most promising because the containment of flow beneath the vehicle results in a high pressure being exerted on the lower surface. Yet a pure waverider with high $L / D$ is always too thin to provide enough volume for fuel and payloads. Typically, the volumetric efficiency of the viscous optimized waverider is lower than 0.12. Although the freestream upper surface of a pure waverider can be morphed to an upwarp to enlarge the volume, but this canopy actually forms a compression surface on the upper part of the vehicle, which leads to an increase in both the aerodynamic drag and the negative lift, causing an overall reduction in $L / D[8,9]$.

To aim at enhancing the aerodynamic performance of hypersonic aircraft with large volume requirements, a new concept called high-pressure capturing wing ( $\mathrm{HCW}$ ) was first proposed by Cui et al. [10]. Unlike generic configurations, a thin wing called the HCW is attached to the top of an upwarp airframe. On the basis of the shock wave compression theory, high-pressure airflow compressed by the upper surface of the vehicle acts on the HCW when flying in the hypersonic regime. Therefore, the aerodynamic lift significantly augments on the vehicle with only a small increase in drag, producing a correspondingly high increase in its $L / D$. Furthermore, such a concept particularly fits for vehicles with large volumes because the lift produced by the HCW increases with the increase of the compression angle in the upwarp.

Expanding on the philosophy of HCWs, a family of novel configurations is proposed in this letter. A typical configuration is shown as Figure 1(a). Note from this figure that there 
are two wings in this configuration. The lower wing is a common compression surface, while the upper wing is designed according to the idea of HCWs. The parts between the two wings are the airframe and the attachment struts. Because the cross-section of the configuration appears like the letter "I", it is thus named "hypersonic I-shaped aerodynamic configuration (HIAC)".

In order to validate the advantages of HIACs, a scaled test model was generated as a typical example. Moreover, the leading edge profiles of both the low wing and the HCW were optimized by using the computational fluid dynamics, the design of experiments method, the surrogate models method, and the genetic algorithm in combination. The optimized configuration is shown as Figure 1(a), and some key geometric parameters of the model are as follows. The total length, the wingspan, and the height of the model are 500, 300 , and $131 \mathrm{~mm}$, respectively. The thickness of the low wing is $5 \mathrm{~mm}$, and the blunt radius on the nose is $2.5 \mathrm{~mm}$. The leading edges of both the HCW and the low wing are defined by quadric curves. The volume $V$ and the normal projection area of the model $S_{\mathrm{p}}$ are $0.00248 \mathrm{~m}^{3}$ and $0.1027 \mathrm{~m}^{2}$, respectively. Accordingly, the volumetric efficiency (defined as $V^{2 / 3} / S_{\mathrm{p}}$ ) is about 0.175 . Besides, the configuration without the HCW and attachment struts is used as a reference model for comparison, as shown in Figure 1(b))

Subsequently, a numerical simulation work was carried out to evaluate the aerodynamic performances of the model. Numerical solutions are obtained by solving the 3-dimensional compressible, Navier-Stokes equations with the use of a second order TVD finite-volume scheme for spatial discretization, a second order implicit time marching scheme, and a realizable $k-\varepsilon$ model that is used in the computations. A full three-dimensional structural grid is used to discretize the computational domain, in which algebraic transfinite interpolation methods with elliptic interior point refinement are utilized. In addition, an overset grid system is employed for ease of generating grids at the expense of interpolation in the overlapping regions. Total mesh number is about 23 million. All meshes near the wall are refined in order to capture the boundary layer. Besides, a grid convergence test has been conducted to ensure the calculation accuracy.

Freestream Mach numbers of 5, 6, and 7, and angles of attack ranging from $-2^{\circ}$ to $12^{\circ}$ are used as flow conditions. A set of typical wind tunnel parameters are taken as flow conditions. Respective values of the parameters are listed in Table 1 , where $M a$ is the Mach number, $P_{0}$ is the total pressure, $T_{0}$ is the total temperature, $R e$ denotes the Reynolds number, and $P_{\infty}$ and $T_{\infty}$ are the static pressure and the static temperature, respectively.

Curves of the $L / D$ versus $C_{\mathrm{L}}$ at each Mach number are plotted in Figure 1(c)-(e). Results of both the HIAC (labeled by "HIAC") and the reference model (labeled as "Ref") are presented for ease of comparison. The reference area for calculating aerodynamic force coefficients is the normal projection area of the model $\left(S_{\mathrm{p}}\right)$. Note from these figures that the variation of the $L / D$ for both models at three Mach numbers is small. This indicates that the $L / D$ values of the models are not sensitive to the Mach number.
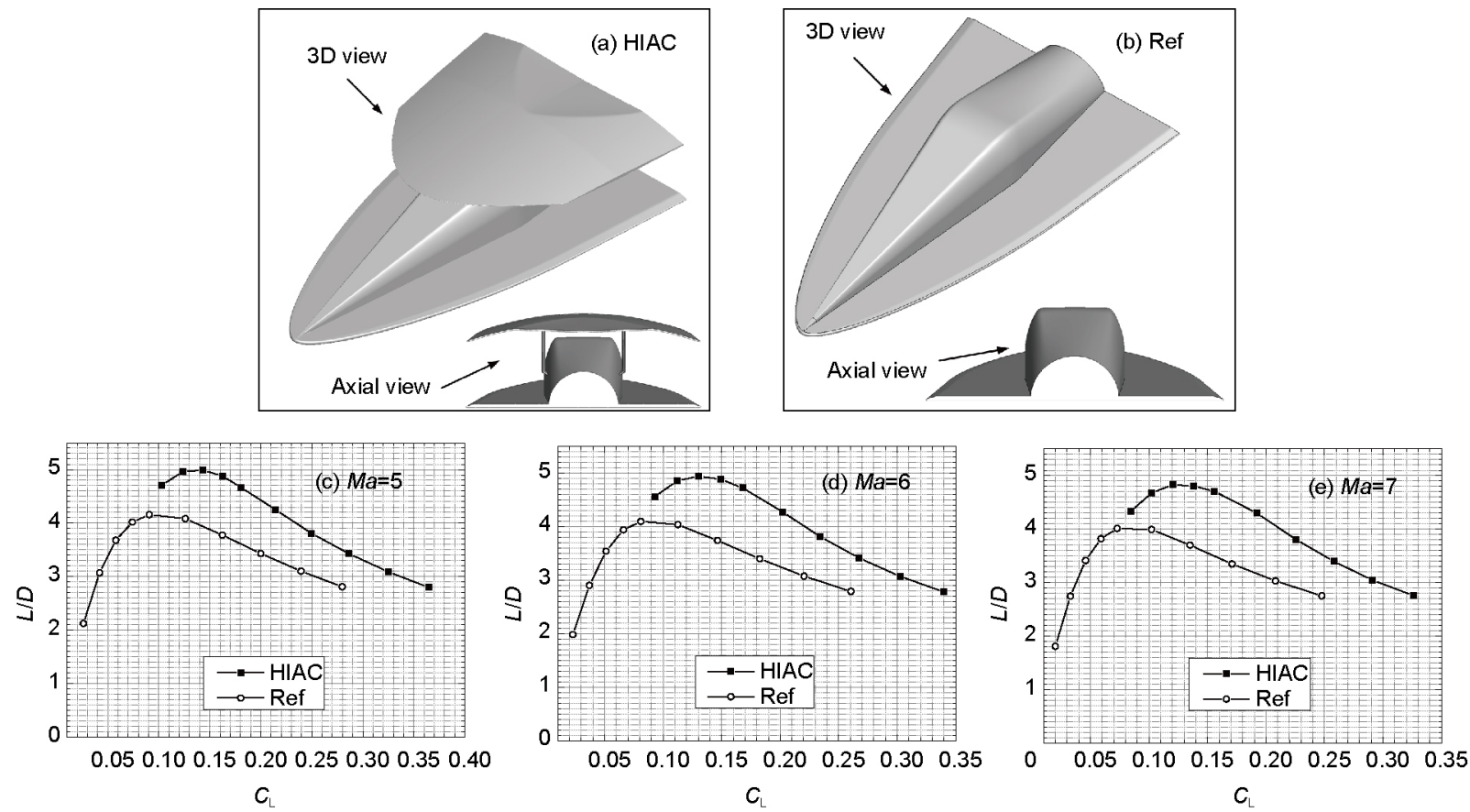

Figure 1 Configuration and aerodynamic performance comparison between the HIAC and the reference model. (a) HIAC; (b) Ref; (c) $M a=5$; (d) $M a=6$; (e) $M a=7$. 
Table 1 Freestream conditions of numerical simulations

\begin{tabular}{|c|c|c|c|c|c|}
\hline$M a$ & $P_{0}(\mathrm{~Pa})$ & $T_{0}(\mathrm{~K})$ & $P_{\infty}(\mathrm{Pa})$ & $T_{\infty}(\mathrm{K})$ & $\operatorname{Re}\left(\mathrm{m}^{-1}\right)$ \\
\hline 5 & $1.0 \times 10^{6}$ & 345 & $1.89 \times 10^{3}$ & 58 & $2.299 \times 10^{7}$ \\
\hline 6 & $2.5 \times 10^{6}$ & 465 & $1.58 \times 10^{3}$ & 57 & $2.365 \times 10^{7}$ \\
\hline
\end{tabular}

In addition, the results also show that although the maximal $L / D$ of the reference model holds relatively high values (about 4.16, 4.09, and 4.01 at Mach number 5, 6, and 7, respectively) because the model has been optimized for several rounds, the maximal $L / D$ (about 4.99, 4.94, and 4.83 at Mach number 5, 6 , and 7, respectively) of the HIAC model improves drastically benefiting from the $\mathrm{HCW}$. The increasing percentage of the maximal $L / D$ is about $20 \%$ at all Mach numbers. Furthermore, the lift coefficients of the HIAC model are substantially higher than those of the reference model at all Mach numbers, the increased percentages $\Delta$ are about $58 \%, 61 \%$, and $65 \%$ at Mach number 5, 6, and 7, respectively. Here the increased percentage $\Delta$ is calculated by

$\Delta=\frac{C_{\mathrm{L}, \mathrm{HIAC}}-C_{\mathrm{L}, \mathrm{Ref}}}{C_{\mathrm{L}, \mathrm{Ref}}}$.

In summary, a new family of hypersonic I-shaped aerodynamic configurations, derived from the high-pressure capturing wing concept, is proposed in this letter. Moreover, the advantages of high $L / D$, high volumetric efficiency, and high lift are clearly demonstrated. In the present study, only the profiles of the leading edges were taken as design variables of the optimization. The aerodynamic performances of the configuration may be further enhanced if the surface shape of the HCW is considered as optimization variables. We believe our present study will promote further research in the aerody- namic design of high-speed configurations, which may ultimately offer a new candidate for hypersonic flight vehicles.

This work was supported by the National Natural Science Foundation of China (Grant Nos. 11372324, and 11572333), and the State Key Laboratory of High Temperature Gas Dynamics, Institute of Mechanics, Chinese Academy of Sciences (Grant Nos. LHD2017TC01, LHD2017MS04, and LHD2017QN03).

1 Z. Chen, C. P. Yu, L. Li, and X. L. Li, Sci. China-Phys. Mech. Astron. 59, 664702 (2016).

2 Q. Wang, J. P. Li, W. Zhao, and Z. L. Jiang, Sci. China-Phys. Mech. Astron. 59, 624701 (2016).

3 H. Zhu, and S. Fu, Sci. China-Phys. Mech. Astron. 60, 104712 (2017).

4 S. Tan, and Q. B. Li, Sci. China-Phys. Mech. Astron. 60, 114713 (2017).

5 K. Cui, S. C. Hu, G. L. Li, Z. P. Qu, and M. Situ, Sci. China Tech. Sci. 56, 1980 (2013).

6 D. Küchemann, The Aerodynamic Design of Aircraft (AIAA Inc., Blacksburg, Virginia, 2012), p.12.

7 M. A. Lobbia, in Optimization of waverider-derived crew reentry vehicles using a rapid aerodynamics analysis approach: Proceedings of the 53rd AIAA Aerospace Sciences Meeting, (Kissimmee, Florida, 2015).

8 K. Kontogiannis, A. Sóbester, and N. Taylor, J. Aircraft 54, 890 (2017).

9 K. Cui, and G. W. Yang, Chin. Sci. Bull. 52, 57 (2007).

10 K. Cui, G. L. Li, Y. Xiao, and Y. Z. Xu, AIAA J. 55, 1909 (2017). 